\title{
Describe molecules by a heterogeneous graph neural network with transformer-like attentions for supervised property predictions
}

Daiguo Deng ${ }^{1}$, Zengrong Lei ${ }^{1}$, Xiaobin Hong ${ }^{1}$, Ruochi Zhang ${ }^{1,3, *}$, Fengfeng Zhou $^{2, *}$.

1 Fermion Technology Co., Ltd, Guangzhou, Guangdong 510000, P.R. China,

2 College of Computer Science and Technology, and Key Laboratory of Symbolic Computation and Knowledge Engineering of Ministry of Education, Jilin University, Changchun, Jilin 130012, P.R. China.

3 School of Artificial Intelligence, Jilin University, Changchun 130012, P.R. China.

* Correspondence may be addressed to Fengfeng Zhou: FengfengZhou@gmail.com or ffzhou@jlu.edu.cn . Lab web site: http://www.healthinformaticslab.org/ . Phone: +86-431-85166024. Fax: +86-431-8516-6024. Correspondence may also be addressed to Ruochi Zhang: zrc720@gmail.com . 
Table S1. The number of parameters in different setting of HiddenState and LayerNum. The column HiddenState represents the dimension of the hidden states of a given model. The column LayerNum is the number of graph layers. The NumParam is the total number of the MolHGT parameters.

\begin{tabular}{|l|l|l|l|l|l|}
\hline HiddenState & LayerNum & NumParam & HiddenState & LayerNum & NumParam \\
\hline 200 & 2 & 3461643 & 520 & 2 & 23137132 \\
\hline 200 & 3 & 5035293 & 520 & 3 & 33708542 \\
\hline 200 & 4 & 6608943 & 520 & 4 & 44279952 \\
\hline 240 & 2 & 4968392 & 560 & 2 & 26821941 \\
\hline 240 & 3 & 7229962 & 560 & 3 & 39079671 \\
\hline 240 & 4 & 9491532 & 560 & 4 & 51337401 \\
\hline 280 & 2 & 6747421 & 600 & 2 & 30778129 \\
\hline 280 & 3 & 9821711 & 600 & 3 & 44846979 \\
\hline 280 & 4 & 12896001 & 600 & 4 & 58915829 \\
\hline 320 & 2 & 8798249 & 640 & 2 & 35007438 \\
\hline 320 & 3 & 12810059 & 640 & 3 & 51012208 \\
\hline 320 & 4 & 16821869 & 640 & 4 & 67016978 \\
\hline 360 & 2 & 11121778 & 680 & 2 & 39508006 \\
\hline 360 & 3 & 16195908 & 680 & 3 & 57573496 \\
\hline 360 & 4 & 21270038 & 680 & 4 & 75638986 \\
\hline 400 & 2 & 13716986 & 720 & 2 & 44281815 \\
\hline 400 & 3 & 19978236 & 720 & 3 & 64532825 \\
\hline 400 & 4 & 26239486 & 720 & 4 & 84783835 \\
\hline 440 & 2 & 16585015 & 760 & 2 & 49326763 \\
\hline 440 & 3 & 24158185 & 760 & 3 & 71888093 \\
\hline 440 & 4 & 31731355 & 760 & 4 & 94449423 \\
\hline 480 & 2 & 19724603 & 800 & 2 & 54645072 \\
\hline 480 & 3 & 28734493 & 800 & 3 & 79641522 \\
\hline 480 & 4 & 37744383 & 800 & 4 & 104637972 \\
\hline
\end{tabular}

